\title{
La capitalisation comme fausse solution à la sortie de crise
}

Pierre Salama

Résumée: Le système de retraite par répartition doit évoluer compte tenu de la transition démographique. On se dirige petit à petit vers un système mixte alliant le système de répartition - avec des retraites calculées à partir d'un salaire de référence faible - et le système de capitalisation, obligatoire ou non, pour ceux qui désireraient avoir une retraite plus élevée, militaires, juges etc., au détriment des catégories vulnérables.

Mots-clé: Transition demographique. Répartition. Capitalisation. Vulnerabilité.

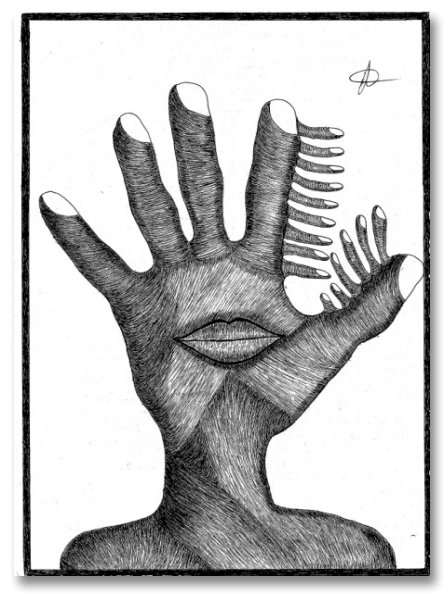

\section{A capitalização como falsa solução para saída da crise}

\section{Pierre Salama}

Resumo: O sistema de aposentadoria deve evoluir à luz da transição demográfica. Estamos gradualmente caminhando para um sistema misto que combina o sistema de distribuição - com pensões calculadas a partir de um salário de referência baixo - e o sistema de capitalização, obrigatório ou não, para aqueles que gostariam de ter uma pensão mais elevada como militares juízes, etc., em detrimento de categorias vulneráveis.

Palavras-chave: Transição demográfica. Distribuição. Capitalização. Vulnerabilidade.

Professeur émérite de l'Université de Paris XIII, CEPN-CNRS UMR 7234, RECEBIDO: 03/07/2019 latino-américaniste.

APROVADO: 07/08/2019

E-mail: psalama@wanadoo.fr 


\section{Introduction}

L'Amérique latine, et aujourd'hui plus particulièrement le Brésil et l'Argentine, connaissent soit un ralentissement de leur croissance soit une profonde crise. La crise que connait l'Argentine est très importante par son ampleur et des effets sociaux, mais aussi parce qu'elle met à nu les échecs de la politique de libéralisation menée par le président Macri. La crise traversée par le Brésil (2015-2016) est la plus importante depuis celle des années 1930, les inégalités et la pauvreté ont augmenté considérablement, effaçant les quelques améliorations obtenues lors des présidences de Lula et la première présidence de Dilma.

La nouvelle présidence brésilienne hérite une situation économique contrastée: de bons fondamentaux, mais une situation sociale très détériorée et une insertion internationale problématique avec une incapacité à rebondir après la crise de 2015 - 2016. Fin 2018, certains fondamentaux sont positifs: un faible déficit de la balance des comptes courants : - 0,7 du PIB; un solde primaire du budget (c'est-à-dire sans le service de la dette publique) de 2,3\% du PIB en baisse depuis la crise masquant cependant un déficit nominal encore très élevé: - 7.3\% du PIB à cause du poids du service de la dette; un taux d'inflation modéré $(3,75 \%$ pour l'IPCA, l'indice des prix à la consommation); des réserves internationales élevées (375 milliards de dollars) qui s'expliquent essentiellement par les entrées de capitaux dont surtout les investissements étrangers directs (79 milliards en 2018). Les aspects négatifs sont principalement une croissance poussive: 1,1\% en 2018 (après 1,1\% en 2017) alors qu'on aurait pu attendre une vive reprise après la crise de 2015-2016: - 3,6 et - 3,5\% respectivement du chute du PIB, un taux de chômage à deux chiffres, une précarité croissante avec un déclassement et une désaffiliation en hausse, un endettement public important, une incapacité croissante à s'insérer positivement dans la division internationale du travail. 2019 devait consacrée une forte croissance. Chaque jour qui passe montre que cet espoir est vain, la croissance sera bien plus faible que celle prévue.

Le courant libéral considère que l'excès d'impôt et de cotisations produit nécessairement un effet d'éviction sur l'épargne destinée à l'investissement. Dit autrement le fait de payer des impôts prive 
ceux qui désireraient investir de pouvoir le faire. La diminution de la pression fiscale devrait donc inciter à investir. Cette relation tient plus du mythe que de l'analyse sérieuse. Les dépenses publiques, financée par les recettes fiscales et les cotisations, favorisent les économies externes, ont des effets multiplicateurs qui peuvent produire une meilleure rentabilisation dont profiterait le secteur privé, assurent un minimum de solidarité intergénérationnel quand le système de retraite repose sur le système de répartition de nature à réduire la pauvreté et légèrement les inégalités de revenus. A l'inverse, l'épargne augmentée de la diminution de la pression fiscale, ne se dirige pas nécessairement vers l'investissement productif. Elle peut être destinée à l'achat de bien de consommation et surtout à a des placements financiers.

Pour autant, ce sont celles que propose la présidence de Bolsanaro. Entres autres mesures, passer d'un système de répartition à demain u système de capitalisation serait la solution pour sortir de la crise et retrouver et la confiance des marchés, et une croissance élevée.

Cet article a pour objet d'analyser les raisons pour lesquels le système de retraite en Amérique latine doit évoluer compte tenu de la transition démographique sans pour autant entrer dans un système de capitalisation lorsqu'existent des systèmes de répartition.

\section{Du passé-présent...}

Ces quarante dernières années se caractérisent par une tendance à la stagnation du PIB par tête et une désindustrialisation dans la plupart des pays latino-américains (CASTILLO; MARTINS, 2016; SALAMA, 2014; RODRIK, 2016; MONCAYO JIMENEZ, 2013). Contrairement à une idée relativement partagée ces économies ont été peu ou pas émergentes, à l'exception de la première décennie des années 2000. Elles n'ont pas convergé ou peu vers le niveau de revenu par tête des pays avancés, contrairement à de nombreux pays asiatiques. Le Brésil, pays emblématique à la fois par son poids économique, par le rayonnement de la politique menée par le président Lula (2003 - 2011), par le résultat des dernières élections 
présidentielles conduisant l'extrême droite au pouvoir dès 2019, ne connait pas cette convergence. Son PIB par tête, mesuré à l'aune de celui des Etats-Unis, ne converge pas. Il est approximativement le même qu'en 1960, même si dans les années 1960 et dans la première décennie des années 2000 il s'en est rapproché. La Corée du sud dès le début des années 1990, croise le Brésil, révélant ainsi que la tendance à la stagnation n'est pas une fatalité.

Figure 1 : Revenu par tête du Brésil et de la Corée du sud, 1960-2016 De 1960-20̄16.

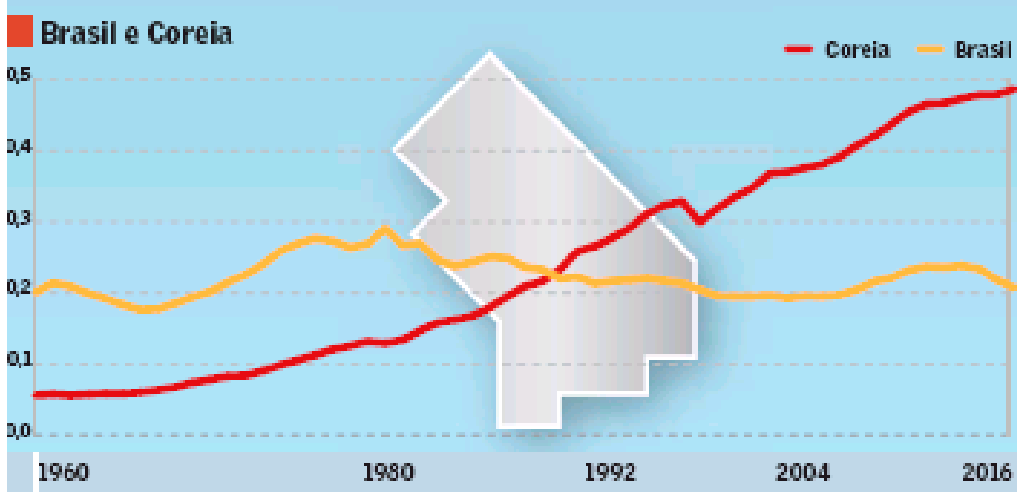

Source: Valor Econômico, 13 dez. 2018.

L'amélioration du niveau de vie des nouvelles classes moyennes-basses ou moyennes alimentait au début des années 2000 un certain optimisme sur leur parcours de vie, les conduisant à être plus «tolérante» lorsque le revenu des classes moyennes supérieures et riches augmentait plus rapidement que le leur. C'est ce que Hirschman nommait l'effet "tunnel» (HIRSCHMAN; ROTHSCHILD, 1973). Ce n'est plus le cas depuis 2014 au Brésil et dans de nombreux autres pays. La tolérance devient intolérance avec à la fois l'accentuation de la reprimarisation de l'économie, la poursuite de la désindustrialisation et la crise dès 2015 - 2016.

Avec la reprimarisation de l'économie et consécutivement la désindustrialisation, la demande de travail qualifié proposée par les entreprises est ainsi devenue relativement plus rare, en deça de l'offre des jeunes issus de l'école et des universités. En d'autres 
termes, la reprimarisation a favorisé la création d'emplois non qualifiés alors même que la qualification augmentait, d'où un déclassement réel et ressenti encore plus vivement par les jeunes accédant au statut des classes moyennes. Les données sont éloquentes: 38\% des salariés ayant suivi un enseignement supérieur complet occupent des emplois moins qualifiés que ceux auxquels ils auraient pu prétendre, ce pourcentage s'élève à $44 \%$ pour la tranche d'âge de 24 à 35 ans. Ces pourcentages étaient inférieurs en 2012: 33,4\% et 38,4\% respectivement au Brésil (O Valor 13 dec. 2018, selon les données de l'IPEA et de la PNAD) (SALAMA, 2012; ROCHA, 2014; AMARANTE; COLACCE, 2018). La baisse des inégalités des revenus du travail peut cacher une réduction relative le la part des revenus des classes moyennes-basses et moyennes.

L'Amérique latine et plus particulièrement le Brésil n'ont pas connu de miracle économique. La reprimarisation de leurs économies a entrainé une plus grande vulnérabilité et a accentué une désindustrialisation précoce. La pauvreté a diminué - il s'agit d'une mesure en terme absolue, ce qui signifie qu'elle aurait pu disparaitre - mais les revenus relatifs des couches moyennes-basses et moyennes ont baissé, suscitant à terme une frustration. Les catégories les plus riches se sont enrichies et quand la crise est arrivée, les partis progressistes ont été d'autant plus facilement désignés comme boucs émissaires qu'ils avaient niés l'enrichissement des plus riches et l'appauvrissement relatif d'une grande partie des couches moyennes, et qu'ils étaient atteints, à l'égal des autres partis, par la gangrène de la corruption. Ne pas comprendre ces mouvements souterrains n'a pas permis de leur faire face lorsqu'ils sont apparus au grand jour.

\section{3 ... au présent- futur}

Ce que nous souhaiterions ici analyser ici est une des tendances lourdes, non pas que ce soit la seule, mais elle est importante. Il s'agit de l'évolution démographique et ses effets potentiels sur les systèmes de retraite vers davantage de capitalisation et moins de répartition, ce dernier système tendant à se convertir en revenu universel minimal pour les personnes âgées. 
La transition démographique a été particulièrement rapide en Amérique latine. De ce fait, ses effets se font sentir plus rapidement que dans les pays avancés. La baisse des taux bruts de natalité et de mortalité devraient conduire à une augmentation de la population à un rythme de plus en plus faible et à une inversion de celle-ci et donc à une réduction de la population dès 2045, tout au moins au Brésil, pays le plus important du sous continent américain.

\section{Figure 2 : Brésil, population, taux brut de natalité, taux brut de mortalité, 1950-2095}

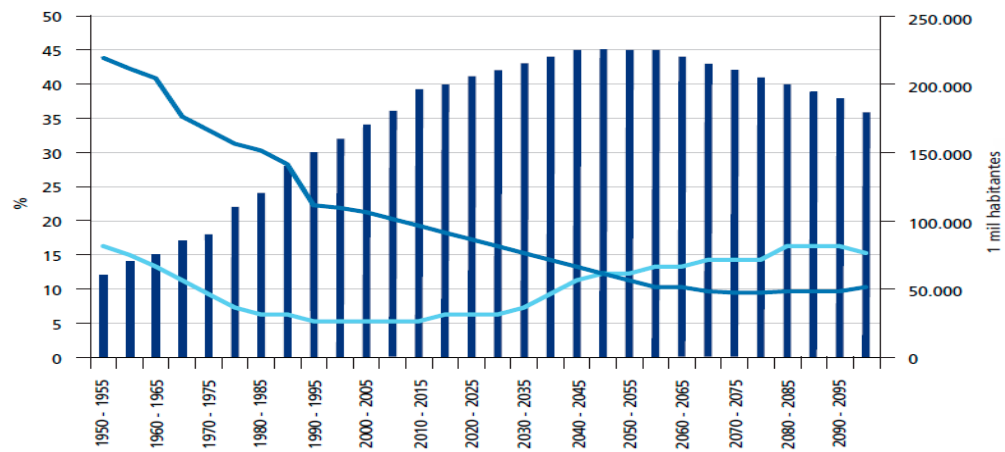

Source: Guellati, Monteiro et Oliveira Jr (2017).

Avec la généralisation de l'éducation en Amérique latine ${ }^{2}$, le taux de fécondité des femmes a baissé rapidement ${ }^{3}$. Avec l'amélioration des soins, la mortalité infantile a baissé et l'espérance de vie a augmenté, moins rapidement certes pour les catégories les plus pauvres de la population mais au total de manière significative.

1 Nombre d'enfants (de morts) sur population totale pour une année calendaire. 2 Le nombre d'années en moyenne de scolarité était au Brésil en 1980 de 5.7 années, il s'élève à 8.2 années en 2015. Au Chili à cette date, il atteint 9.7 années et en Argentine 9.2 années. Le pourcentage d'enfants de 15 à 18 ans ayant accompli un enseignement élémentaire complet au Brésil passe entre 1980 et 2010 de 48\% à 69.4\%. Voir Guellati, Monteiro et Oliveira Jr. (2017).

3 Selon les Nation Unies, le nombre d'enfants par femme est passé en moyenne en Amérique latine de 6 entre 1960-1965 à un peu plus de 2 entre 2000-2015 et devrait se situer autour de 1.8 entre 2030-2035. (UN, 2015). Pour le Brésil, selon Guellati, Monteiro et Oliveira Jr. (2017), à partir des de données de l'IBGE, le taux de fécondité total des femmes en âge de procréer est passé de 6.3 en 1960 à 1.9 en 2010 et devrait se situer à 1.7 en 2020. Tant en Amérique latine qu'au Brésil, la transition démographique est donc particulièrement rapide et si la population continue à croitre, à un rythme de plus en plus faible, cela vient de ce que la génération en âge de procréer correspond au taux de fécondité plus élevé qu'au temps t existant vingt ans auparavant. 
Aujourd'hui, ces données s'approchent de celles observées dans les pays avancés comme on peut le voir dans le tableau 2.

\section{Tableau 1: Principaux indicateurs démographiques de quelques pays latino-américains, 2014}

\begin{tabular}{|l|l|l|l|l|l|l|}
\hline Pays & $\begin{array}{l}\text { Population } \\
\text { (en millions) }\end{array}$ & $\begin{array}{l}65 \text { ans et } \\
\text { plus en \% }\end{array}$ & $\begin{array}{l}\text { Ratio de } \\
\text { dépendance* }\end{array}$ & $\begin{array}{l}\text { Esperance } \\
\text { de vie des } \\
\text { hommes }\end{array}$ & $\begin{array}{l}\text { Esperance } \\
\text { de vie des } \\
\text { femmes }\end{array}$ & $\begin{array}{l}\text { PIB/t (PPA, } \\
\text { 2015) en \$ }\end{array}$ \\
\hline Argentine & 43 & 10.8 & 56.6 & 72.3 & 79.9 & 20.364 \\
\hline Bolivie & 10.6 & 6.3 & 64.6 & 65.5 & 70.4 & 6.954 \\
\hline Brésil & 206.1 & 7.6 & 45.1 & 70.4 & 78 & 15.391 \\
\hline Chili & 17.8 & 10.7 & 45.3 & 78.8 & 84.2 & 23.367 \\
\hline Colombie & 47.8 & 6.8 & 45.9 & 70.3 & 77.5 & 13.829 \\
\hline Equateur & 15.9 & 6.5 & 55.9 & 72.9 & 78.5 & 8.620 \\
\hline Mexique & 125.4 & 6.3 & 52.4 & 74.2 & 79 & 16.988 \\
\hline
\end{tabular}

Source: Figliuli (2018, p. 30). * ratio entre la population âgée de 0-14 ans plus de 65 ans sur celle âgés de 15-64 ans.

\section{Figure 3: Population âgé de plus de 60 ans en Amérique latine et} dans le monde, 2015-2100

\section{2015}

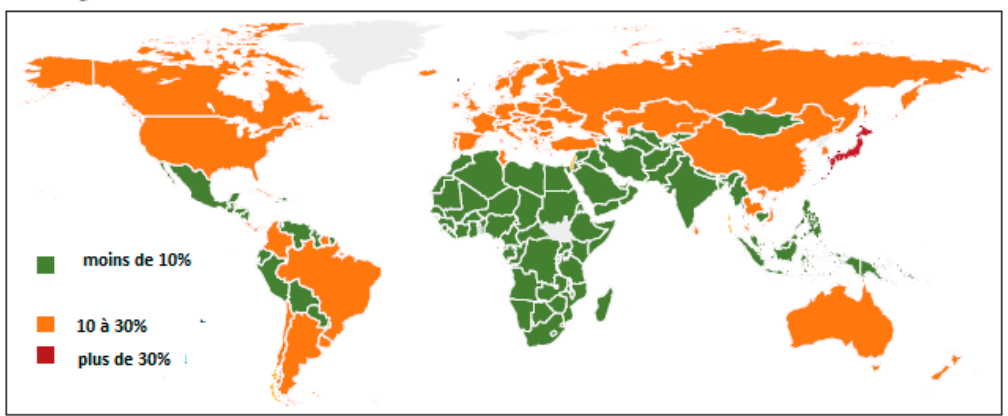

\section{2100}

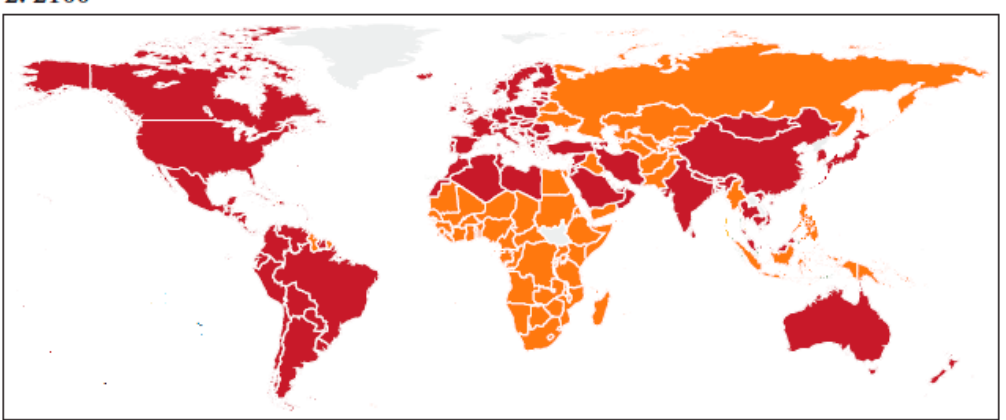

Source : Figliuli (2018, p.16). 
Tableau 2 : Dépenses en santé publique en Amérique latine, quelques pays, en pourcentage du PIB, 2015 -2065

\begin{tabular}{|l|c|c|c|}
\hline Pays & 2015 & 2030 & 2065 \\
\hline Argentine & 6.1 & 7.4 & 12.6 \\
\hline Bolivie & 4.3 & 5.4 & 9.4 \\
\hline Brésil & 4.6 & 6.3 & 12 \\
\hline Chili & 3.8 & 5.2 & 9.5 \\
\hline Colombie & 5.5 & 7.6 & 14.3 \\
\hline Equateur & 3 & 3.9 & 7.2 \\
\hline Mexique & 3.3 & 4.4 & 8.7 \\
\hline
\end{tabular}

Source: Figliuli (2018).

Compte-tenu de l'évolution prévisible des dépenses de santé (tableau 2) et des principaux indicateurs démographiques, on peut considérer que le ratio de dépendance (population de moins de 15 ans plus population de plus de 64 ans sur population de 15 à 64 ans) devrait passer en Amérique latine de 95\% en 1960 à 50\% en 2025 en Amérique latine et Caraïbes pour remonter ensuite selon le rapport des Nations Unies (UN, 2015). D'ici à 2025, il y aura donc de plus en plus d'inactifs pour de moins en moins d'actifs. La remontée à partir de 2025 s'explique par le ralentissement du vieillissement de la population et de la baisse du taux de fécondité. Les conséquences économiques sont à la fois une augmentation inexorable du poids des retraites et des frais de santé dans le PIB, quel que soient les systèmes, publics-privés - assistancialiste (sans contribution) pour la santé, répartition - capitalisation, mixte, assistancialiste (sans contribution) ou non pour les retraites.

En ce qui concerne les retraites, le système de répartition privilégie la solidarité intergénérationnelle, les actifs payant pour les inactifs. Le système de capitalisation privilégie l'engagement individuel, l'actif, et parfois l'entrepreneur qui l'emploie si la capitalisation est obligatoire. Le produit des cotisations est placé dans un fonds de pension le met sur le marché financier. Les systèmes mixtes existent dans de nombreux pays (répartition concentrée dans la fonction publique, plus capitalisation). Comme les emplois informels sont très importants et qu'en conséquence le taux de couverture pourrait être faible si on tenait compte des seules cotisations, de nombreux pays pays ont mis en place, à des degrés divers, des retraites minimales pour ceux qui n'auraient pu 
contribuer en raison de la faiblesse de leurs revenus liés à l'informalité de leurs emplois.

Ainsi qu'on peut le voir, les taux de couverture sont très différents (figure 4). Plus de 50\% de la population ne reçoit pas de retraites en 2016 au Venezuela (données de 2013), au Paraguay, en Colombie (données de 2013), en République dominicaine et dans la plupart des pays d'Amérique centrale. A l'inverse, le taux de couverture est particulièrement important en Bolivie, avec une part non contributive considérable. Il est important dans les autres pays, plus de 70\% (à l'exception du Pérou et de l'Equateur) avec une part non contributive importante au Mexique, au Chili.

\section{Figure 4: Taux de couverture, contribution (répartition) et non contribution, 2016}

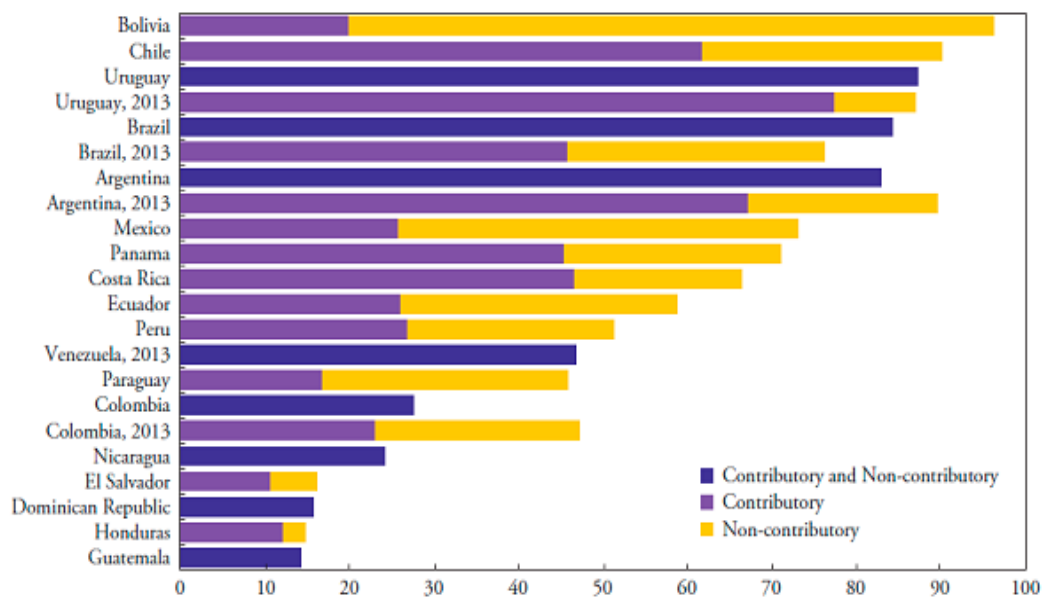

Source: Figliuli (2018, p. 39).

Pensions payées par l'Etat (avec et sans cotisations) en \% du PIB de 2015 à 2065, quelques pays, 2014

\begin{tabular}{|l|l|l|l|l|}
\hline Pays & Système de retraite & 2015 & 2030 & 2065 \\
\hline Argentine & $\mathrm{R}$ & 7.8 & 8.3 & 13.8 \\
\hline Bolivie & $\mathrm{C}$ & 3 & 1.9 & 2.4 \\
\hline Brésil & $\mathrm{R}$ & 11.2 & 17.1 & 29.5 \\
\hline Chili & $\mathrm{C}$ & 4.1 & 3 & 4.8 \\
\hline Colombie & R et C & 5.1 & 4.1 & 3 \\
\hline Equateur & $\mathrm{R}$ & 2.7 & 3.5 & 6.4 \\
\hline Mexique & $\mathrm{C}$ & 1.7 & 2.3 & 1.8 \\
\hline
\end{tabular}

Source : Figliuli $(2018$, p. 46$){ }^{*}$ C: capitalisation, R: répartition, M: mixte. 
Ces prévisions ne sont pas sans poser des problèmes économiques appelant à des choix politiques clairs. D'une manière générale, deux options existent. La première consiste à préserver la notion de service public et à considérer que tout n'est pas marchand, ce qui n'exonère pas d'évaluer son coût économique et au besoin de le financer par une réforme fiscale visant à faire principalement payer aux catégories les plus aisées une solidarité sociale permettant de vivre mieux dans une société davantage plus inclusive. La seconde consiste à diminuer fortement la part prise par le système de répartition (augmenter les cotisations, allonger leur durée, diminuer le salaire de référence par rapport auquel est calculée la retraite) en faveur d'un système de capitalisation. Pour ce qui concerne la santé, la tendance est de privilégier de plus en plus appel les hôpitaux privés avec à la clef l'essor des systèmes d'assurance privés, obligatoires ou non. Enfin, pour l'éducation, la tendance est également de privilégier l'enseignement privé dans le primaire et le secondaire - le public étant réservé aux catégories pauvres et modestes - et d'augmenter les droits d'inscription dans les universités publiques. La conséquence est à la fois une débudgétisation de plus en plus importante de ces dépenses sociales, un essor du crédit - et de la financiarisation qui va avec - pour financer partiellement ces dépenses et un endettement consécutif des ménages affectant le revenu disponible surtout des catégories modestes (LAVINAS, 2017). La solidarité liée au service public diminue alors fortement.

Sauf mouvements sociaux de grande ampleur, les pays qui avaient opté pour un système de répartition, se dirigent petit à petit vers un système mixte alliant le système de répartition - avec des retraites calculées à partir d'un salaire de référence faible et le système de capitalisation, obligatoire ou non, pour ceux qui désireraient avoir une retraite plus élevée. Ceux qui n'auraient pu contribuer, parce que pauvres et/ou handicapés, recevraient une retraite minimale proche du maximum de retraite obtenu grâce à la répartition. On aurait ainsi un système de retraite universel par répartition faible couplé d'un système par capitalisation de plus en plus important, avec cependant de nombreuses exceptions probablement concernant les militaires, les juges et quelques 
autres catégories de la fonction publique. A ces exceptions près, la fonction publique dans l'ensemble serait alignée sur le système de retraite du privé (durée de contribution, âge minimale de départ à la retraite, contributions variables visant à réduire voire à éliminer le déficit du système de répartition). Cette réforme rapprocherait les pays ayant opté pour la répartition vers ceux qui comme le Chili, la Colombie, le Mexique ont favorisé le système de capitalisation, dégageant ainsi des ressources pour le secteur financier, en espérant qu'un jour cela favoriserait la croissance... et pas seulement la spéculation boursière.

\section{Conclusion}

Paraphrasant "Los siete locos» de Robert Arlt (1929), sept propositions surréalistes pour éviter contrarier un avenir qui s'annonce difficile pour la majeure partie de la population.

Il existe une "solution surréaliste" alternative aux politiques qu'appliquent les gouvernements face aux crises et aux défis produits par l'évolution de l'économie mondiale. Au lieu de s'ouvrir, alors même que monte le protectionnisme, au lieu d'imposer une régression du pouvoir d'achat au prétexte de retrouver une économie compétitivité sans se poser la question des efforts à faire en terme de productivité, au lieu de poursuivre dans une voie rentière, que celle-ci repose sur l'exploitation des matières premières ou bien sur les transferts massifs provenant des travailleurs migrants aux Etats-Unis, au lieu de favoriser la reprimarisation favorisant les comportements rentiers au détriment de l'industrialisation, les gouvernements latino-américains devraient chercher une rupture "surréaliste" avec les décisions de politique économique passées. Pour cela, je me suis inspiré d'un grand écrivain argentin Roberto Arlt. Dans le roman écrit au début du $\mathrm{XX}^{\circ}$ siècle, "Los siete locos", Artl fait des propositions surréalistes pour sortir de la misère que connaissaient les migrants en Argentine dans les années 1920, un peu comme en France lorsque les manifestants scandaient en mai 1968: "Soyons réalistes, demandons l'impossible". 
Pour sortir de cette situation de crise profonde, il y a sept conditions surréalistes. La première, consiste à repenser la relation entre le marché intérieur et le marché extérieur. La croissance viendra de l'augmentation du pouvoir d'achat des gens, c'est-à-dire, du marché intérieur et non pas du marché extérieur, les économies étant encore relativement fermées. Dans le monde d'aujourd'hui, marché intérieur et marché extérieur constituent un tout organique, favoriser l'un en oubliant l'autre c'est favoriser les importations, se condamner à moyen terme à des dévaluations récessives à coûts sociaux élevés. La croissance retrouvée ne peut exister de manière durable que si la compétitivité augmente et que puisse se développer, de nouveau, le tissu industriel C'est la seconde proposition "surréaliste". La troisième proposition surréaliste vise à rompre avec les politiques destructrices de l'environnement et celles qui limitent les droits sociaux des citoyens. La quatrième proposition surréaliste est d'accroître la recherche développement avec une politique industrielle favorisant les entreprises "porteuses" d'avenir. La cinquième proposition surréaliste consiste à investir massivement dans l'éducation et la recherche; le cinquième, comme l'ont fait les pays asiatiques, chercher un taux de change déprécié face au dollar. La sixième, la plus surréaliste, vise à imposer une réforme fiscale progressive et non plus régressive. Enfin, la septième proposition surréaliste est de favoriser une politique de redistribution en faveur des personnes les plus vulnérables qui ne soit pas conçue comme un devoir de charité mais comme un droit pour les personnes les plus vulnérables.

Telles sont les conditions surréalistes, folles pour certains, mais nécessaires par ce qu'elles entrent en harmonie avec une éthique sociale et parce que les autres ont lamentablement échoué. Comme l'économie ne saurait se réduire à une "boite à outils" et à de simples techniques, ces mesures surréalistes ne peuvent s'imposer que par des mobilisations sociales. La voie est étroite pour éviter de sombrer dans un futur qui déchante, c'est cependant la seule voie à emprunter pour contrarier cet avenir.

Ces mesures sont plus efficaces et éthiquement plus justes que celles qui consistent à mettre en place une réforme profonde du système de répartition pour les retraites basé sur la solidarité 
intergénérationnelle et un système de capitalisation de plus en plus important financé par les salariés et les entreprises, réservé pour l'essentiel aux classes moyennes et aux plus riches. Le raisonnement économique qui justifie que la seule manière de diminuer le déficit public serait de réduire le poids des retraites dans les dépenses publiques et de retrouver ainsi la confiance des marchés et de là, de manière automatique, une croissance perdue est un raisonnement qui ne repose sur aucune base solide. L'exemple du Mexique est là pour le montrer : le taux de croissance moyen de ces vingt dernières années est un des plus faible d'Amérique latine malgré de la mise en place du système de capitalisation comme système principal pour les retraites. Au-delà de la fragilité du raisonnement économique qui sous tend cette proposition de réforme du système de retraite, ce raisonnement repose sur la croyance en une confiance retrouvée obtenu grâce à la réduction du déficit budgétaire, et à celle du poids de l'Etat dans le PIB. Le coût social de cette croyance est élevé. Les mesures surréalistes évoquées ci-dessus sont au contraire de nature à la fois de surmonter les problèmes posés par la démographie et de renouer avec une croissance plus élevée et une mobilité sociale plus importante.

\section{RÉFÉRENCES}

AMARANTE, V.; COLACCCE, M. Mas o menos desigualidades? Una revision sobre la desigualidad de los ingresos a nivel global, regional y nacional. Revista de La Cepal, Santiago de Chile, n. 124, p. 07 - 35, 2018.

ARLT, R. Les sept fous. Paris: Pierre Belfond, 1929.

CASTILLO, M.; MARTINS NETO, A. Premature desindustrialization. Revista de la Cepal, Santiago de Chile, n. 205, p. 01 -23, 2016.

FIGLIULI, L. Growing Pains, is Latin America Prepared for Population Aging? IMF, Western Hemisphere Department, n. 18/05, p. 30, 2018.

GUELLATI, Y.; MONTEIRO, C. D.; OLIVEIRA JR.; A. de. O Brasil em 2035 : tendências e incertezas para a área social. Rio de Janeiro: IPEA, 2017.

HIRSCHMAN, A. O.; ROTHSCHILD, M. The Changing Tolerance for Income Inequality in the Course of Economic Development. The Quarterly Journal of Economics, Oxford, v. 87, n. 4, p. $544-566,1973$. 
LAVINAS, L. The Takeover of Social Policy by Financialisation: the Brazilian Paradox. New York: Palgrave Mc Millan, 2017.

MONCAYO JIMENEZ, E. Cambio estructural, crecimiento y (des) industrialization en América latine, 1950- 2005. Bogotá: Universidad Nacional de Colombia, 2013.

ROCHA, S. Allocations sociales et pauvreté au Brésil. Paris: FMSH, 2014.

RODRIK, D. Premature Deindustrialzation. Journal of Economic Growth, [on line], v. 21, n. 1, p. $01-33,2016$.

SALAMA, P. Des pays toujours émergents?. [s.l.]: La documentation française, 2014.

Les économies émergentes latino-américaines. Paris: Armand Colin, 2012.

UN - United Nations. World population prospects: The 2015 Revision. [s.l.]: [s.n.], 2015. Disponible à: <https://population.un.org/wpp/>. Accès en: 25 oct. 2019. 